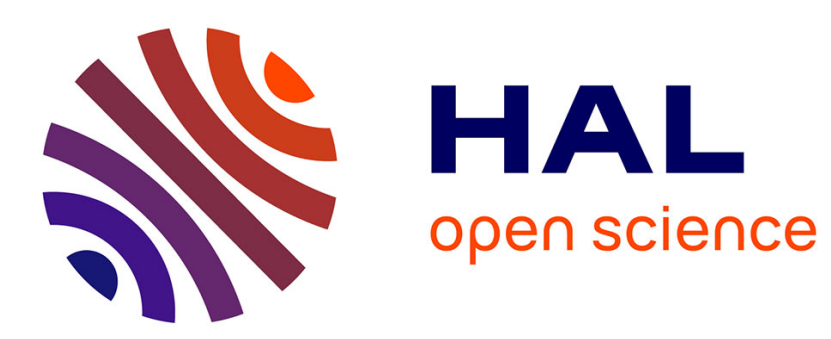

\title{
Sliding mode control of systems with time-varying delays via descriptor approach
}

\author{
E. Fridman, Frédéric Gouaisbaut, Marc Dambrine, J.-P. Richard
}

\section{To cite this version:}

E. Fridman, Frédéric Gouaisbaut, Marc Dambrine, J.-P. Richard. Sliding mode control of systems with time-varying delays via descriptor approach. International Journal of Systems Science, 2003, 34 (8-9), pp.553 - 559. 10.1080/00207720310001609020 . hal-01767598

\section{HAL Id: hal-01767598 \\ https://hal.science/hal-01767598}

Submitted on 16 Apr 2018

HAL is a multi-disciplinary open access archive for the deposit and dissemination of scientific research documents, whether they are published or not. The documents may come from teaching and research institutions in France or abroad, or from public or private research centers.
L'archive ouverte pluridisciplinaire HAL, est destinée au dépôt et à la diffusion de documents scientifiques de niveau recherche, publiés ou non, émanant des établissements d'enseignement et de recherche français ou étrangers, des laboratoires publics ou privés. 


\title{
Sliding mode control of systems with time-varying delays via descriptor approach
}

\author{
E. Fridman $b$, F. Gouaisbaut $\downarrow$, M. Dambrine $\downarrow$ and J.-P. Richard $\downarrow$ \\ b Department of Electrical Engineering-Systems \\ Tel-Aviv University, Tel-Aviv 69978, Israel. \\ Ł LAIL UMR 8021 Ecole Centrale de Lille, 59651 Villeneuve d'ascq cedex,
}

\begin{abstract}
In this paper, we combine a descriptor approach to stability and control of linear systems with time-varying delays, which is based on the Lyapunov - Krasovskii techniques, with a recent result on sliding mode control of such systems. The systems under consideration have norm-bounded uncertainties and uncertain bounded delays. The solution is given in terms of linear matrix inequalities (LMIs) and improves the previous results based on other Lyapunov techniques. A numerical example illustrates the advantages of the new method.
\end{abstract}

\section{Introduction}

The interest in robust control of time-delay systems this last decade is witnessed by the rich dedicated literature (see for instance, [1]- [17] and the numerous references therein). Many existing results concern systems with unknown but constant delays. But in some applications, such as networked control or tele-operated systems, the assumption of a constant delay is too restrictive: this can lead to bad performances or, even worse, to unstability

This paper combines two previous results so to obtain a more efficient sliding mode controller for uncertain systems with time-varying delays and norm-bounded uncertainties. Other results [9] concern varying delays but may lead to strong conditions which reduces the dynamic performances.

The first of these results is the sliding mode design given in [9], which copes with stabilization of systems with time-varying delays. The approach relies on the construction on a LyapunovRazumikhin function which allows fast variations of the delay but leads to some conservatism on the upper bound of the time-delay. 
The second result given in [3] concerns the construction of a new class of Lyapunov-Krasovskii functionals using a descriptor model transformation. Unlike previous transformations, the descriptor model leads to a system which is equivalent to the original one (from the point of view of stability) and requires bounding of fewer cross-terms. Furthermore, following this approach, stability criteria have been given in [6] for systems with time-varying delays without any assumption on their derivatives (which was the case with the usual Lyapunov-Krasovskii functionals).

The paper is organized as follows: In section 2, we develop a Lyapunov-Krasovskii approach on a descriptor representation for an uncertain, linear, time-delay system. This provides a stability condition expressed in term of feasibility of a linear matrix inequality (LMI) (see [1]). Then, the design of a stabilizing memoryless state feedback is derived. Section 3 deals with the design of a sliding mode controller. This is achieved through the resolution of a generalized eigenvalue problem which can be solved efficiently using semi-definite programming tools. In the last section, an illustrative example is solved using our approach and comparison with previous results are provided.

\section{Notation:}

Throughout the paper the superscript ' $T$ ' stands for matrix transposition, $\mathcal{R}^{n}$ denotes the $n$ dimensional Euclidean space, $\mathcal{R}^{n \times m}$ is the set of all $n \times m$ real matrices. The notation $P>0$, for $P \in \mathcal{R}^{n \times n}$ means that $P$ is symmetric and positive definite. $I_{n}$ represents the $n \times n$ identity matrix.

\section{Stabilization of linear systems with norm-bounded un- certainties by delayed feedback}

In this section we consider the following uncertain linear system with a time-varying delay:

$$
\begin{aligned}
& \dot{x}(t)=\left(A_{0}+H \Delta(t) E_{0}\right) x(t)+\left(A_{1}+H \Delta(t) E_{1}\right) x(t-\tau(t))+\left(B_{0}+H \Delta(t) E_{2}\right) u(t)+B_{1} u(t-\tau(t)), \\
& \quad x(t)=\phi(t), t \in[-h, 0],
\end{aligned}
$$

where $x(t) \in \mathcal{R}^{n}$ is the system state, $u(t) \in \mathcal{R}^{m}$ is the control input, $h$ is an upper-bound on the time-delay function $(0 \leq \tau(t) \leq h, \forall t \geq 0)$. The matrix $\Delta(t) \in \mathcal{R}^{p \times q}$ is a matrix of time-varying, uncertain parameters satisfying

$$
\Delta^{T}(t) \Delta(t) \leq I_{q} \forall t
$$

For simplicity, we consider only one delay, but the results of this section may be easily generalized to the case of multiple delays.

We seek a control law

$$
u(t)=K x(t)
$$

that will asymptotically stabilize the system. 


\subsection{The stability issue}

In this subsection, we consider the following equation:

$$
\dot{x}(t)=\left(\bar{A}_{0}+H \Delta(t) \bar{E}_{0}\right) x(t)+\left(\bar{A}_{1}+H \Delta(t) \bar{E}_{1}\right) x(t-\tau(t)) .
$$

Representing (1) in an equivalent descriptor form [3]:

$$
\dot{x}(t)=y(t), \quad 0=-y(t)+\left(\bar{A}_{T}+H \Delta \bar{E}_{T}\right) x(t)-\left(\bar{A}_{1}+H \Delta \bar{E}_{1}\right) \int_{t-\tau(t)}^{t} y(s) d s
$$

or

$$
E \dot{\bar{x}}(t)=\left[\begin{array}{cc}
0 & I_{n} \\
\bar{A}_{T}+H \Delta \bar{E}_{T} & -I_{n}
\end{array}\right] \bar{x}(t)-\left[\begin{array}{c}
0 \\
\bar{A}_{1}+H \Delta \bar{E}_{1}
\end{array}\right] \int_{t-\tau(t)}^{t} y(s) d s,
$$

with

$$
\begin{aligned}
\bar{x}(t) & =\operatorname{col}\{x(t), y(t)\}, E=\operatorname{diag}\left\{I_{n}, 0\right\}, \\
\bar{A}_{T} & =\bar{A}_{0}+\bar{A}_{1}, \bar{E}_{T}=\bar{E}_{0}+\bar{E}_{1},
\end{aligned}
$$

the following Lyapunov-Krasovskii functional is applied:

$$
V(t)=\bar{x}^{T}(t) E P \bar{x}(t)+V_{2}(t),
$$

where

$$
\begin{gathered}
P=\left[\begin{array}{cc}
P_{1} & 0 \\
P_{2} & P_{3}
\end{array}\right], \quad P_{1}>0, \quad E P=P^{T} E \geq 0, \\
V_{2}(t)=\int_{-h}^{0} \int_{t+\theta}^{t} y^{T}(s)\left[R+\delta_{2} \bar{E}_{1}^{T} \bar{E}_{1}\right] y(s) d s d \theta .
\end{gathered}
$$

The following result is obtained:

Lemma 1 The system (4) is asymptotically stable if there exist $n \times n$ matrices $0<P_{1}, P_{2}, P_{3}, R>0$ and positive numbers $\delta_{1}, \delta_{2}$ that satisfy the following LMI:

$$
\Gamma=\left[\begin{array}{cccc}
\Psi & h P^{T}\left[\begin{array}{c}
0 \\
\bar{A}_{1}
\end{array}\right] & P^{T}\left[\begin{array}{c}
0 \\
H
\end{array}\right] & h P^{T}\left[\begin{array}{l}
0 \\
H
\end{array}\right] \\
* & -h R & 0 & 0 \\
* & * & -\delta_{1} I_{p} & 0 \\
* & * & * & -\delta_{2} h I_{p}
\end{array}\right]<0
$$

where

$$
\begin{gathered}
\Psi=\Psi_{0}+\left[\begin{array}{cc}
\delta_{1} \bar{E}_{T}^{T} \bar{E}_{T} & 0 \\
0 & h\left(R+\delta_{2} \bar{E}_{1}^{T} \bar{E}_{1}\right)
\end{array}\right], \\
\Psi_{0}=P^{T}\left[\begin{array}{cc}
0 & I_{n} \\
\bar{A}_{T} & -I_{n}
\end{array}\right]+\left[\begin{array}{cc}
0 & I_{n} \\
\bar{A}_{T} & -I_{n}
\end{array}\right]^{T} P,
\end{gathered}
$$

and $*$ denotes symmetrical entries. 
Proof. Note that

$$
\bar{x}^{T}(t) E P \bar{x}(t)=x^{T}(t) P_{1} x(t)
$$

and, hence, differentiating the first term of (6) with respect to $t$ gives:

$$
\frac{d}{d t}\left\{\bar{x}^{T}(t) E P \bar{x}(t)\right\}=2 x^{T}(t) P_{1} \dot{x}(t)=2 \bar{x}^{T}(t) P^{T}\left[\begin{array}{c}
\dot{x}(t) \\
0
\end{array}\right] .
$$

Replacing $\left[\begin{array}{c}\dot{x}(t) \\ 0\end{array}\right]$ by the right side of (5) we obtain:

$$
\frac{d V(t)}{d t}=\bar{x}^{T}(t) \Psi_{0} \bar{x}(t)+\eta_{0}+\eta_{1}+\eta_{2}+h y^{T}(t)\left[R+\delta_{2} \bar{E}_{1}^{T} \bar{E}_{1}\right] y(t)-\int_{t-h}^{t} y^{T}(s)\left[R+\delta_{2} \bar{E}_{1}^{T} \bar{E}_{1}\right] y(s) d s
$$

where

$$
\begin{gathered}
\eta_{0}(t) \triangleq-2 \int_{t-\tau(t)}^{t} \bar{x}^{T}(t) P^{T}\left[\begin{array}{c}
0 \\
\bar{A}_{1}
\end{array}\right] y(s) d s, \\
\eta_{1}(t) \triangleq 2 \bar{x}^{T}(t) P^{T}\left[\begin{array}{r}
0 \\
H
\end{array}\right] \Delta\left(\bar{E}_{0}+\bar{E}_{1}\right) x(t), \\
\eta_{2}(t) \triangleq-2 \int_{t-\tau(t)}^{t} \bar{x}^{T}(t) P^{T}\left[\begin{array}{c}
0 \\
H
\end{array}\right] \Delta \bar{E}_{1} y(s) d s .
\end{gathered}
$$

Applying the standard bounding

$$
a^{T} b \leq a^{T} R a+b^{T} R^{-1} b, \quad \forall a, b \in \mathcal{R}^{n}, \forall R \in \mathcal{R}^{n \times n}: R>0,
$$

and using the fact that $\tau(t) \leq h$, we have

$$
\begin{aligned}
& \eta_{0}(t) \leq \tau \bar{x}^{T}(t) P^{T}\left[\begin{array}{c}
0 \\
\bar{A}_{1}
\end{array}\right] R^{-1}\left[0 \bar{A}_{1}^{T}\right] P \bar{x}(t)+\int_{t-\tau(t)}^{t} y^{T}(s) R y(s) d s \\
& \leq h \bar{x}^{T}(t) P^{T}\left[\begin{array}{c}
0 \\
\bar{A}_{1}
\end{array}\right] R^{-1}\left[\begin{array}{ll}
0 & \left.\bar{A}_{1}^{T}\right] P \bar{x}(t)+\int_{t-h}^{t} y^{T}(s) R y(s) d s .
\end{array}\right.
\end{aligned}
$$

Similarly

$$
\begin{gathered}
\eta_{1} \leq \delta_{1}^{-1} \bar{x}^{T}(t) P^{T}\left[\begin{array}{r}
0 \\
H
\end{array}\right]\left[0 H^{T}\right] P \bar{x}(t)+\delta_{1} x^{T}(t) \bar{E}_{T}^{T} \bar{E}_{T} x(t), \\
\eta_{2} \leq h \delta_{2}^{-1} \bar{x}^{T}(t) P^{T}\left[\begin{array}{r}
0 \\
H
\end{array}\right]\left[0 H^{T}\right] P \bar{x}(t)+\delta_{2} \int_{t-h}^{t} y^{T}(s) \bar{E}_{1}^{T} \bar{E}_{1} y(s) d s .
\end{gathered}
$$

Substituting the right sides of the latter inequalities into (10), we obtain

$$
\frac{d V(t)}{d t} \leq \bar{x}^{T}(t) \bar{\Gamma} \bar{x}(t)
$$


where

$$
\bar{\Gamma}=\Psi+h P^{T}\left[\begin{array}{c}
0 \\
\bar{A}_{1}
\end{array}\right] R^{-1}\left[0 \bar{A}_{1}^{T}\right] P+\left(\delta_{1}^{-1}+h \delta_{2}^{-1}\right) P^{T}\left[\begin{array}{c}
0 \\
H
\end{array}\right]\left[0 H^{T}\right] P .
$$

Therefore, LMI (8) yields by Schur complements that $\bar{\Gamma}<0$ and hence $\dot{V}<0$, while $V \geq 0$, and thus (4) is asymptotically stable [13], [4].

\subsection{State-feedback stabilization}

The results of Lemma 1 can also be used to verify the stability of the closed-loop obtained by applying (3) to the system (1) if we set in (8)

$$
\bar{A}_{i}=A_{i}+B_{i} K, i=0,1, \quad \bar{E}_{0}=E_{0}+E_{2} K
$$

and verify that the resulting LMI is feasible. The problem with (8) is that it is linear in its variables only when the state-feedback gain $K$ is given. In order to find $K$ we apply again Schur formula to $\bar{\Gamma}$, the $\Psi$ term being expanded. We thus obtain the following matrix inequality:

$$
\left[\begin{array}{ccccccc}
\Psi_{0} & h P^{T}\left[\begin{array}{c}
0 \\
\bar{A}_{1} R^{-1}
\end{array}\right] & {\left[\begin{array}{c}
0 \\
h I_{n}
\end{array}\right]} & {\left[\begin{array}{c}
\bar{E}_{T}^{T} \\
0
\end{array}\right]} & h\left[\begin{array}{c}
0 \\
\bar{E}_{1}^{T}
\end{array}\right] & \delta_{1}^{-1} P^{T}\left[\begin{array}{c}
0 \\
H
\end{array}\right] & \left.\delta_{2}^{-1} h P^{T}\left[\begin{array}{c}
0 \\
H
\end{array}\right]\right] \\
* & -h R^{-1} & 0 & 0 & 0 & 0 & 0 \\
* & * & -h R^{-1} & 0 & 0 & 0 & 0 \\
* & * & * & -\delta_{1}^{-1} I_{q} & 0 & 0 & 0 \\
* & * & * & * & -\delta_{2}^{-1} h I_{q} & 0 & 0 \\
* & * & * & * & * & -\delta_{1}^{-1} I_{p} & 0 \\
* & * & * & * & * & * & -\delta_{2}^{-1} h I_{p}
\end{array}\right]<0
$$

Consider the inverse of $P$. It is obvious, from the requirement $P_{1}>0$ and the fact that in (8) $-\left(P_{3}+P_{3}^{T}\right)$ must be negative definite, that $P$ is nonsingular. Defining

$$
P^{-1}=Q=\left[\begin{array}{cc}
Q_{1} & 0 \\
Q_{2} & Q_{3}
\end{array}\right] \text { and } M=\operatorname{diag}\left\{Q, I_{2(n+p+q)}\right\}
$$

we multiply (14) by $M^{T}$ and $M$, on the left and on the right, respectively. Choosing

$$
R^{-1}=Q_{1} \varepsilon
$$


where $\varepsilon$ is a positive number, and introducing $\bar{\delta}_{1}=\delta_{1}^{-1}$ and $\bar{\delta}_{2}=\delta_{2}^{-1}$, we obtain the LMI

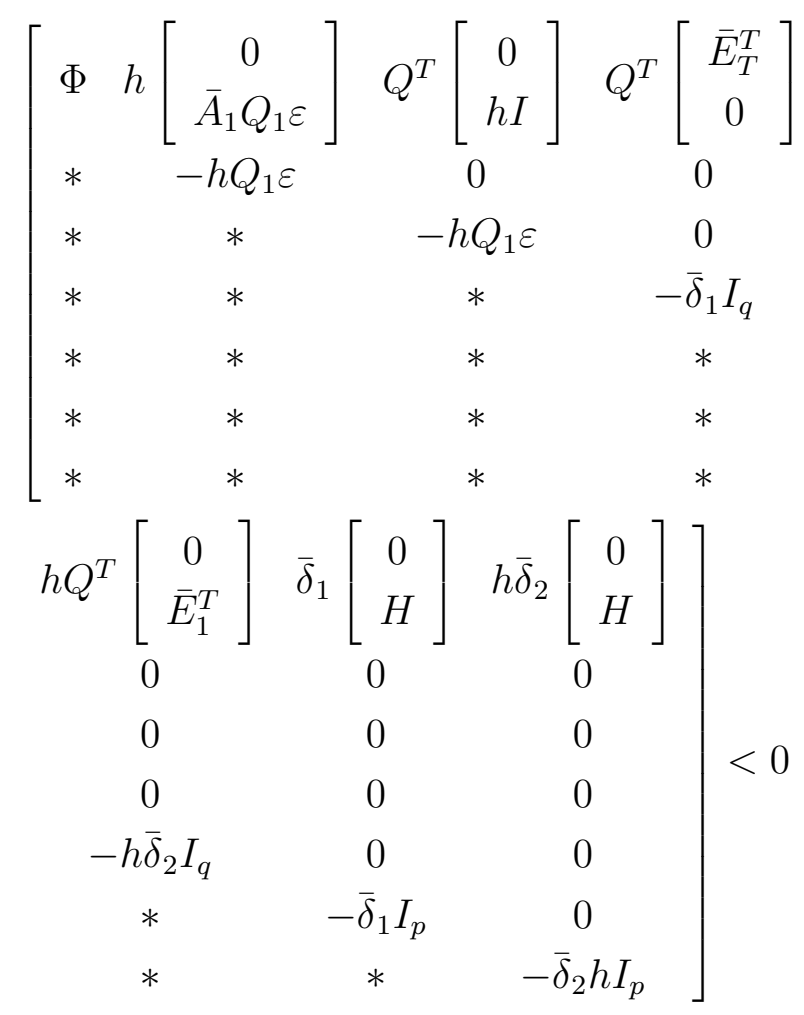

where

$$
\Phi=\left[\begin{array}{cc}
0 & I_{n} \\
\bar{A}_{T} & -I_{n}
\end{array}\right] Q+Q^{T}\left[\begin{array}{cc}
0 & I_{n} \\
\bar{A}_{T} & -I_{n}
\end{array}\right]^{T} .
$$

Substituting (13) into (16) and denoting $Y=K Q_{1}, B_{T}=B_{0}+B_{1}$, we obtain

Theorem 1 The control law of (3) asymptotically stabilizes (1) if, for some positive number $\varepsilon$, there exist scalars $\bar{\delta}_{1}>0, \bar{\delta}_{2}>0$ and matrices $0<Q_{1}, Q_{2}, Q_{3}, \in \mathcal{R}^{n \times n} Y \in \mathcal{R}^{m \times n}$ that satisfy the following LMI:

$$
\left[\begin{array}{cccc}
Q_{2}+Q_{2}^{T} & Q_{1} A_{T}^{T}+Y^{T} B_{T}^{T}-Q_{2}^{T}+Q_{3} & 0 & h Q_{2}^{T} \\
* & -Q_{3}-Q_{3}^{T} & h \varepsilon\left(A_{1} Q_{1}+B_{1} Y\right) & h Q_{3}^{T} \\
* & * & -h \varepsilon Q_{1} & 0 \\
* & * & * & -h Q_{1} \varepsilon \\
* & * & * & * \\
* & * & * & * \\
* & * & * & * \\
* & * & * & *
\end{array}\right.
$$




$$
\left.\begin{array}{cccc}
Q_{1} E_{T}^{T}+Y^{T} E_{2}^{T} & h Q_{2}^{T} E_{1}^{T} & 0 & 0 \\
0 & h Q_{3}^{T} E_{1}^{T} & \bar{\delta}_{1} H & h \bar{\delta}_{2} H \\
0 & 0 & 0 & 0 \\
0 & 0 & 0 & 0 \\
-\bar{\delta}_{1} I_{q} & 0 & 0 & 0 \\
* & -h \bar{\delta}_{2} I_{q} & 0 & 0 \\
* & * & -\bar{\delta}_{1} I_{p} & 0 \\
* & * & * & -\bar{\delta}_{2} h I_{p}
\end{array}\right]<0
$$

The state-feedback gain is then given by

$$
K=Y Q_{1}^{-1}
$$

\section{Sliding mode controller}

In this section, we focus on time-delay systems that can be represented, possibly, after a change of state coordinates and input, in the following regular form $([9],[18])$ :

$$
\left\{\begin{aligned}
& \frac{d z_{1}(t)}{d t}=\left(A_{11}+H \Delta(t) E_{0}\right) z_{1}(t)+\left(A_{d 11}+H \Delta(t) E_{1}\right) z_{1}(t-\tau(t)) \\
&+\left(A_{12}+H \Delta(t) E_{2}\right) z_{2}(t)+A_{d 12} z_{2}(t-\tau(t)) \\
& \frac{d z_{2}(t)}{d t}= \sum_{i=1}^{2}\left(A_{2 i} z_{i}(t)+A_{d 2 i} z_{i}(t-\tau)\right)+D u(t)+f\left(t, z_{t}\right) \\
& z(t)=\phi(t) \text { for } t \in[-h, 0]
\end{aligned}\right.
$$

where $z(t)=\left(z_{1}, z_{2}\right)^{T}, z_{1} \in \mathcal{R}^{n-m}, z_{2} \in \mathcal{R}^{m}, A_{i j}, A_{d i j}, i=1,2, j=1,2, E_{k}, k=0,1,2, H$ are constant matrices of appropriate dimensions, $D$ is a regular $m \times m$ matrix, the matrix $\Delta(t)$ is a time-varying matrix of uncertain parameters, $u \in \mathcal{R}^{m}$ is the input vector, $\tau$ is time-varying delay satisfying $0 \leq \tau(t) \leq h, \forall t \geq 0, z_{t}(\theta)$ is the function associated with $z$ and defined on $[-h, 0]$ by $z_{t}(\theta)=z(t+\theta), \phi$ is the initial piecewise continuous function defined on $[-h, 0]$.

We will assume that:

A1) $\left(A_{11}+A_{d 11}, A_{12}+A_{d 12}\right)$ is controllable.

A2) $f$ is Lipschitz continuous and satisfies the inequality

$$
\left\|f\left(t, z_{t}\right)\right\|<F_{M}\left(t, z_{t}\right), \quad \forall t \geq 0,
$$

where $F_{M}\left(t, z_{t}\right)$ is a continuous functional assumed to be known a priori,

A3) $\Delta(t)$ is a time-varying matrix of uncertain parameters satisfying $\Delta^{T}(t) \Delta(t) \leq I \forall t$.

Consider the following switching function:

$$
s(z)=z_{2}-K z_{1}
$$


with $K \in \mathcal{R}^{m \times(n-m)}$. Let $\Omega, \Theta$ be the linear functions defined by

$$
\begin{gathered}
\Omega(z(t))=\sum_{i=1}^{2}\left(A_{2 i}-K A_{1 i}\right) z_{i}(t), \\
\Theta(z(t))=E_{0} z_{1}(t)+E_{2} z_{2}(t)
\end{gathered}
$$

and let $D_{M}$ be the following functional:

$$
\begin{aligned}
D_{M}\left(z_{t}\right)= & \left(\left\|A_{d 21}-K A_{d 11}\right\|+\|K H\|\left\|E_{1}\right\|\right) \sup _{-h \leq \theta \leq 0}\left\|z_{1}(t+\theta)\right\| \\
& +\left\|A_{d 22}-K A_{d 12}\right\| \sup _{-h \leq \theta \leq 0}\left\|z_{2}(t+\theta)\right\| .
\end{aligned}
$$

Following [9] and using the results of previous section, we are able to design a sliding mode controller that will stabilize system (20) under less conservative assumptions on the delay law.

Theorem 2 Assume A1-A3. If, for some positive number $\varepsilon$, there exist positive numbers $\bar{\delta}_{1}, \bar{\delta}_{2}$ and matrices $0<Q_{1}, Q_{2}, Q_{3} \in \mathcal{R}^{(n-m) \times(n-m)}, Y \in \mathcal{R}^{m \times(n-m)}$ that satisfy the following LMI:

$$
\begin{aligned}
& {\left[\begin{array}{cccc}
Q_{2}+Q_{2}^{T} & X_{12} & 0 & h Q_{2}^{T} \\
* & -Q_{3}-Q_{3}^{T} & h \varepsilon\left(A_{d 11} Q_{1}+A_{d 12} Y\right) & h Q_{3}^{T} \\
* & * & -h \varepsilon Q_{1} & 0 \\
* & * & * & -h \varepsilon Q_{1} \\
* & * & * & * \\
* & * & * & * \\
* & * & * & * \\
* & * & * & *
\end{array}\right.} \\
& \left.\begin{array}{cccc}
Q_{1} E_{T}^{T}+Y^{T} E_{2}^{T} & h Q_{2}^{T} E_{1}^{T} & 0 & 0 \\
0 & h Q_{3}^{T} E_{1}^{T} & \bar{\delta}_{1} H & h \bar{\delta}_{2} H \\
0 & 0 & 0 & 0 \\
0 & 0 & 0 & 0 \\
-\bar{\delta}_{1} I & 0 & 0 & 0 \\
* & -h \bar{\delta}_{2} I & 0 & 0 \\
* & * & -\bar{\delta}_{1} I & 0 \\
* & * & * & -\bar{\delta}_{2} h I
\end{array}\right]<0 .
\end{aligned}
$$

where

$$
X_{12}=Q_{1}\left(A_{11}^{T}+A_{d 11}^{T}\right)+Y^{T}\left(A_{12}^{T}+A_{d 12}^{T}\right)-Q_{2}^{T}+Q_{3},
$$

then the sliding mode control law

$$
u(t)=-D^{-1}\left[\Omega(z(t))+\left(F_{M}\left(t, z_{t}\right)+D_{M}\left(z_{t}\right)+\|K H\|\|\Theta(z(t))\|+M\right) \frac{s(z(t))}{\|s(z(t))\|}\right],
$$

where $K=Y Q_{1}^{-1}, M>0$ and $s, \Omega, \Theta, D_{M}$ are defined in (21)-(23), asymptotically stabilizes system (20) for any delay function $\tau(t) \leq h$. 
Proof : The proof is divided into two parts. The first one is dedicated to the proof of the existence of an ideal sliding motion on the surface $s(z)=0$, the second part to the proof of the stability of the reduced system.

\section{Attractivity of the manifold:}

Consider the Lyapunov-Krasovskii functional

$$
V(t)=s^{T}(z(t)) s(z(t))=\|s(z(t))\|^{2} .
$$

Differentiating (26) on the trajectories of the closed-loop system gives

$$
\begin{aligned}
\dot{V}(t)= & 2 s^{T}(t)\left(\Omega(z(t))+\sum_{i=1}^{2}\left[A_{d 2 i}-K A_{d 1 i}\right] z_{i}(t-\tau)+D u(t)+\right. \\
& \left.f\left(t, z_{t}\right)-K H \Delta(t)\left[\Theta(z(t))+E_{1} z_{1}(t-\tau(t))\right]\right),
\end{aligned}
$$

Using the expression of the control law (25), we get

$$
\begin{aligned}
\dot{V}(t)= & 2 s^{T}(t)\left(\sum_{i=1}^{2}\left(A_{d 2 i}-K A_{d 1 i}\right) z_{i}(t-\tau)+f\left(t, z_{t}\right)-K H \Delta(t)\left[\Theta(z(t))+E_{1} z_{1}(t-\tau(t))\right]-\right. \\
& {\left.\left[F_{M}\left(t, z_{t}\right)+D_{M}\left(z_{t}\right)+\|K H\|\|\Theta(z(t))\|+M\right] \frac{s}{\|s\|}\right) }
\end{aligned}
$$

then we derive that:

$$
\dot{V} \leq-2 M \| s\left(z(t) \|=-2 M V(t)^{\frac{1}{2}} .\right.
$$

This last inequality is known to prove the finite-time convergence of the system (20) into the surface $s=0([18])$.

\section{Stability of the reduced system:}

On the sliding manifold $s(z)=0$, the system is driven by the following reduced system:

$$
\frac{d z_{1}(t)}{d t}=\left(A_{11}+A_{12} K+H \Delta(t)\left(E_{0}+E_{2} K\right)\right) z_{1}(t)+\left(A_{d 11}+A_{d 12} K+H \Delta(t) E_{1}\right) z_{1}(t-\tau(t))
$$

According to Theorem 1, this system is asymptotically stable for any delay law $\tau(t) \leq h$ if, for some positive number $\varepsilon$, there exist positive numbers $\bar{\delta}_{1}, \bar{\delta}_{2}$ and matrices $0<Q_{1}, Q_{2}, Q_{3}, Y \in \mathcal{R}^{m \times(n-m)}$ that satisfy the LMI (24).

Remark 1 Note that the explicit knowledge of the time-dependance of the delay is not required in the expression of the control law $u(t)$, all is needed is the knowledge of an upper bound $h$.

\section{Example}

We demonstrate the applicability of the above theory by solving the example from [9] for a system without uncertainty. Consider system

$$
\dot{x}(t)=A x(t)+A_{d} x(t-\tau)+B[u(t)+f(x, t)],
$$




\begin{tabular}{|l|l|l|}
\hline & delay upper bound & type of delay \\
\hline Theorem 2 & 3.999 & time-varying \\
\hline Gouaisbaut et al [9] & 1.65 & constant \\
\hline Ivanescu et al.[10] & 1.46 & constant \\
\hline Fu et al.[8] & 0.984 & constant \\
\hline Li and de Souza[14] & 0.51 & constant \\
\hline
\end{tabular}

Table 1: Comparison of results for example (27)-(28)

with a time-varying delay, where

$$
A=\left[\begin{array}{cc}
2 & 0 \\
1.75 & 0.25
\end{array}\right], A_{d}=\left[\begin{array}{cc}
-1 & 0 \\
-0.1 & -0.25
\end{array}\right], B=\left[\begin{array}{l}
1 \\
1
\end{array}\right]
$$

By an appropriate change of variables, this system is equivalent to:

$$
\dot{z}(t)=\tilde{A} z(t)+\tilde{A}_{d} z(t-\tau)+\tilde{B}[u(t)+f(x, t)]
$$

where

$$
\tilde{A}=\left[\begin{array}{ll}
0.25 & 0 \\
1.75 & 2
\end{array}\right], \tilde{A}_{d}=\left[\begin{array}{ll}
-0.9 & -0.65 \\
-0.1 & -0.35
\end{array}\right], \tilde{B}=\left[\begin{array}{l}
0 \\
1
\end{array}\right] .
$$

As the pair $\left(\tilde{A}_{11}, \tilde{A}_{12}\right)$ is not controllable, the system cannot be stabilized independently of the delay.

For this system, previous published works give the following results:

- In the case of a constant delay and $f=0$, the system may be stabilized using a linear memoryless controller $u(t)=K x(t)$ for the following maximum values of $h$ : $h=0.51$ by [14], $h=0.984$ by [8] and $h=1.46$ by [10]. By sliding mode control for the case of constant delay and $f \neq 0$ the maximum value of $h=1.65$.

- Applying Theorem 2 in the case of a time-varying delay and $f \neq 0$, the corresponding value of $h=3.999$ is achieved.

This is summarized in table 1.

\section{Conclusions}

The problem of finding a sliding mode controller that asymptotically stabilizes a system with timevarying delay and norm-bounded uncertainty has been solved. A delay-dependent solution has been derived using a special Lyapunov-Krasovskii functional. The result is based on a sufficient condition 
and it thus entails an overdesign. This overdesign is considerably reduced due to the fact that the method is based on the descriptor representation. As a byproduct for the first time on the basis of the descriptor model transformation the solution to the stabilization problem by the feedback, which depends on both, non-delayed and delayed state is solved. Finally, a numerical example shows the effectiveness of the combined method: sliding mode and descriptor representation.

\section{References}

[1] S. Boyd, L. El Ghaoui, E. Feron, and V. Balakrishnan, 1994, Linear matrix inequality in systems and control theory (SIAM Frontier Series)

[2] L. Dugard and E.I. Verriest (Eds.), 1998, Stability and control of time-delay systems (Springer Verlag)

[3] E. Fridman, 2001, New Lyapunov-Krasovskii functionals for stability of linear retarded and neutral type systems, Systems \& Control Letters, 43, pp. 309-319.

[4] E. Fridman, 2002, Stability of linear descriptor systems with delay: A Lyapunov-based approach, Journal of Mathematical Analysis and Applications, 273, (1), pp. 24-44.

[5] E. Fridman and U. Shaked, 2002, A descriptor system approach to $H_{\infty}$ control of linear timedelay systems. IEEE Transactions on Automatic Control, 47, (2), pp. 253-270.

[6] E. Fridman and U. Shaked, 2003, Delay dependent stability and $H_{\infty}$ control: Constant and time-varying delays. International Journal of Control, 76, (1), 48-60.

[7] E. Fridman and U. Shaked, 2002, An improved stabilization method for linear time-delay systems. IEEE Transactions on Automatic Control, 47, (11),1931- 1937.

[8] M. Fu, H. Li and S.I. Niculescu, 1997, Robust stability and stabilisation of time-delay system via integral quadratic constraint approach. In LNCIS Springer-Verlag, Vol 228, pp 101-116.

[9] F. Gouaisbaut, M. Dambrine and J.P. Richard, 2002, Robust control of delay systems: a sliding mode control design via LMI. Systems \& Control Letters, 46, (4), 219-230.

[10] D. Ivanescu, 2000, Sur la stabilisation des systèmes à retard : théorie et applications. $P h D$ Thesis in Institut National Polytechnique de Grenoble.

[11] V. Kolmanovskii and J-P. Richard, 1999, Stability of some linear systems with delays, IEEE Transactions on Automatic Control, 44, (5), 984-989. 
[12] V. Kolmanovskii, S.I. Niculescu and J. P. Richard, 1999, On the Liapunov-Krasovskii functionals for stability analysis of linear delay systems. International Journal of Control, 72, 374-384.

[13] V. Kolmanovskii and A. Myshkis, 1999, Applied theory of functional differential equations (Kluwer).

[14] X. Li, C. de Souza, 1997, Criteria for robust stability and stabilization of uncertain linear systems with state delay. Automatica, 33, pp. 1657-1662.

[15] M. Mahmoud, 2000, Robust control and filtering for time-delay systems (Marcel Dekker, NewYork).

[16] Y. S. Moon, P. Park, W. H. Kwon and Y. S. Lee, 2001, Delay-dependent robust stabilization of uncertain state-delayed systems. International Journal of Control, 74, pp. 1447-1455.

[17] S-I. Niculescu, 2001: Delay effects on stability: a robust control approach (Springer Verlag)

[18] W. Perruquetti and J-P. Barbot (Eds.), 2002, Sliding mode control in engineering (Marcel Dekker, New-York).

[19] J-P. Richard, 2003: Time delay Systems: An overview of some recent advances and open problems, Automatica, 39 (9), (to be published). 